\title{
Incidence, characteristics, and outcomes of delirium in patients with noninvasive ventilation: a prospective observational study
}

\author{
Rui Zhang, Linfu Bai, Xiaoli Han, Shicong Huang, Lintong Zhou and Jun Duan* (10
}

\begin{abstract}
Background: Factors that may increase the risk for delirium and the firm knowledge around mechanism for delirium in noninvasive ventilation (NIV) patients is lacking. We investigated the incidence, characteristics, and outcomes of delirium in NIV patients.

Methods: A prospective observational study was performed in an intensive care unit (ICU) of a teaching hospital. Patients in whom NIV was used as a first-line intervention were enrolled. During NIV intervention, delirium was screened using the Confusion Assessment Method for the ICU each day. The association between delirium and poor outcomes (e.g., NIV failure, ICU and hospital mortality) was investigated using forward stepwise multivariate logistic regression analyses.

Results: We enrolled 1083 patients. Of these, 196 patients (18.1\%) experienced delirium during NIV intervention. Patients with delirium had higher NIV failure rates (37.8\% vs. $21.0 \%, p<0.01)$, higher ICU mortality (33.2\% vs. 14.3\%, $p<0.01)$, and higher hospital mortality $(37.2 \%$ vs. $17.0 \%, p<0.01)$ than subjects without delirium. They also had a longer duration of NIV (median 6.3 vs. 3.7 days, $p<0.01$ ), and stayed longer in the ICU (median 9.0 vs. 6.0 days, $p<0.01$ ) and the hospital (median 14.5 vs. 11.0 days, $p<0.01$ ). These results were confirmed in COPD and non-COPD cohorts. According to subtype, compared to hyperactive delirium patients, hypoactive and mixed delirium patients spent more days and many more days on NIV (median 3.4 vs. 6.5 vs. 10.1 days, $p<0.01$ ). Similar outcomes were found for length of stay in the ICU and hospital. However, NIV failure, ICU mortality, and hospital mortality did not differ among the three subtypes.
\end{abstract}

Conclusions: Delirium is associated with increases in poor outcomes (NIV failure, ICU mortality, and hospital mortality) and the use of medical resources (duration of NIV, and lengths of stay in the ICU and hospital). Regarding subtype, hypoactive and mixed delirium are associated with higher, and much higher, consumption of medical resources, respectively, compared to hyperactive delirium.

Keywords: Delirium, Noninvasive ventilation, Risk factor

*Correspondence: duanjun412589@163.com

Department of Respiratory and Critical Care Medicine, The First Affiliated

Hospital of Chongqing Medical University, Youyi Road 1, Yuzhong

District, Chongqing 400016, China

\begin{abstract}
Background
Delirium is characterized by acute onset of cerebral dysfunction with a change or fluctuation in baseline mental status, inattention, and either disorganized thinking or altered consciousness [1]. Delirium is divided into hyperactive, hypoactive, and mixed subtypes [2]. It frequently occurs in critically ill patients due to effects of their
\end{abstract}


acute illness as well as pain, stress, anxiety, sleep deprivation, sedation, analgesia, and antimicrobial exposure [3-7]. And its pathophysiology has not yet been clearly established.

A systematic review and meta-analysis reported that the pooled incidence of delirium is $31.8 \%$ in critically ill patients [8]; it is reportedly much higher (reaching 80\%) in mechanically ventilated patients [9]. Delirium is associated with prolonged length of stay (LOS) in the ICU and the hospital, increased hospital mortality, and elevated hospital costs [8, 10-12]. Many strategies to prevent delirium have been investigated [13-15]. However, such studies have generally focused on mechanically ventilated or surgical patients. Knowledge of delirium in patients who have received noninvasive ventilation (NIV) is lacking.

In this study, we investigated the incidence, clinical characteristics, and outcomes of delirium in NIV patients, as well as the distribution of its different subtypes and the association between delirium and NIV failure.

\section{Methods \\ Patients}

This was a prospective observational study performed in an ICU of a teaching hospital from 2016 to 2018. The study protocol was approved by the hospital's institutional review board. Due to the observational nature of this study, informed consent was waived. Patients who were admitted to the ICU for NIV as a first-line intervention were enrolled, and patients younger than 18 years old were excluded. Patients who were treated with NIV after high-flow nasal cannula or invasive mechanical ventilation were also excluded.

\section{NIV management}

NIV (BiPAP Vision or V60; Philips Respironics, Carlsbad, CA) was initiated by the attending physicians in relation to previously described indications [16, 17]. In patients with chronic obstructive pulmonary disease (COPD), the indications for NIV were respiratory rate more than 25 breaths/min, $\mathrm{PaCO}_{2}$ more than $45 \mathrm{mmHg}$, $\mathrm{pH}$ less than $7.35, \mathrm{PaO}_{2} / \mathrm{FiO}_{2}$ less than $200 \mathrm{mmHg}$, and vigorous activity of the accessory respiratory muscles. In patients with hypoxemic respiratory failure, the indications for NIV were clinical presentation of respiratory distress at rest (such as active contraction of the accessory inspiratory muscles or paradoxical abdominal motion) and $\mathrm{PaO}_{2} /$ $\mathrm{FiO}_{2}$ less than $300 \mathrm{mmHg}$.

A face mask (ZS-MZ-A Face Mask, Shanghai Zhongshan Medical Technology Co., Shanghai, China) was the first choice to connect the patient to the ventilator. The size of the mask was chosen to fit the patients' face type. The S/T mode was used in COPD patients or other patients with labored breathing. The initial inspiratory positive airway pressure was $8-10 \mathrm{cmH}_{2} \mathrm{O}$, which was gradually increased to reach a tidal volume of $6-8 \mathrm{~mL} /$ $\mathrm{kg}$ or the maximal tolerated level. The initial expiratory positive airway pressure was $4 \mathrm{cmH}_{2} \mathrm{O}$. This was gradually increased to counterbalance the intrinsic positive end expiratory pressure in COPD patients and titrated according to the flow curve to ensure that expiratory flow reached zero prior to inspiration or diminished ineffective efforts. In patients with hypoxemic respiratory failure, it was increased to maintain alveoli patency and to elevate the end expiratory lung volume. In patients with heart failure, continuous positive airway pressure was used if the $\mathrm{PaCO}_{2}$ less than $45 \mathrm{mmHg}$ and $\mathrm{S} / \mathrm{T}$ was used if $\mathrm{PaCO}_{2}$ more than $45 \mathrm{mmHg}$. In all patients, the ventilator parameters were also adjusted according to the actual $\mathrm{PaCO}_{2}$ and $\mathrm{PaO}_{2}$. The fractional concentration of oxygen was set to reach a peripheral oxygen saturation of $>92 \%$.

At the beginning of the treatment, continuous use of NIV was encouraged. Once the patient recovered from respiratory failure, liberation from NIV was considered, following our hospital protocols [18]. However, intubation was indicated if respiratory failure progressively deteriorated. The criteria for intubation were as follows: respiratory or cardiac arrest, failure to maintain $\mathrm{PaO}_{2} /$ $\mathrm{FiO}_{2}$ more than 100, development of a condition necessitating intubation to protect the airway (coma or seizure disorder) or to manage copious tracheal secretions, inability to correct dyspnea, lack of improvement of signs of respiratory muscle fatigue, and hemodynamic instability without response to fluids and vasoactive agents $[16,17]$. NIV failure was defined as requirement for intubation.

\section{Assessment of delirium}

Delirium was screened using the Confusion Assessment Method for the ICU (CAM-ICU) every morning from NIV initiation to termination [9]. The assessment was performed by trained researchers. Delirium was assessed based on the following four features: (1) fluctuation in mental status, (2) inattention, (3) disorganized thinking, and (4) altered consciousness. Delirium was diagnosed in the presence of features 1 and 2 and either feature 3 or 4 . The level of consciousness was assessed using the Richmond Agitation-Sedation Scale (RASS) [19]. RASS is a 10-point scale ranging from unarousable ( -5 points) through calm ( 0 points) to combative ( 4 points). The three subtypes of delirium were defined as follows [20]. Hyperactive delirium was defined as present in patients with all positive daily RASS scores (range, 1-4 points) associated with every positive CAM-ICU assessment. Hypoactive delirium was defined as present in patients with all neutral or negative daily RASS scores (range, 0 
to -3 points) associated with every positive CAM-ICU assessment. Mixed delirium was defined as present in patients with daily RASS scores that included both positive values (range, 1-4 points) and neutral or negative values (range, 0 to -3 points) associated with every positive CAM-ICU assessment.

\section{Outcomes}

The primary outcome was the rate of NIV failure. The second outcome included ICU mortality, hospital mortality, duration of NIV, length of stay in the ICU, length of stay in the hospital, and delirium days. The data was collected before hospital discharge. If patient died during hospitalization, the data was collected at the day of death.

\section{Statistical analyses}

Statistical software (SPSS 17.0; IBM Corp., Armonk, NY) was used to analyze the data. Continuous variables are presented as means with standard deviations or medians and interquartile ranges (25-75\%) when appropriate. The normality of data distribution was analyzed using the Shapiro-Wilk test. Differences between groups were analyzed using the Student's t test or Mann-Whitney U test as appropriate. Categorical variables are reported as frequencies and percentages. Differences between groups were analyzed using the chi-square test or Fisher's exact test. Variables with $p<0.1$, as computed in univariate analyses, and other clinical meaningful variables were entered into forward stepwise multivariate logistic regression analyses to identify the association between delirium and poor outcomes (e.g., NIV failure, ICU mortality, and hospital mortality). Bootstrap (1000 samples) was used to validate the association between delirium and poor outcomes. In addition, Cox regression analyses were also performed to retest the association between delirium and poor outcomes. Because delirium can occur at any time during NIV intervention, it was considered to be a time-varying covariate. The time was limited to 65 days as the longest duration of NIV was 65 day. The probability that patients would remain on NIV and in the ICU was analyzed using Kaplan-Meier curves (log-rank test). The threshold for statistical significance was set to $p<0.05$.

\section{Results}

We enrolled 1083 patients in the study. Of these, 196 (18.1\%) developed delirium during NIV intervention. There were no differences between patients with and without delirium in reason for NIV, underlying disease, sex, heart rate, respiratory rate, $\mathrm{PaCO}_{2}$, and $\mathrm{PaO}_{2} / \mathrm{FiO}_{2}$ (Table 1). However, delirious patients were older than non-delirious ones, and they also had higher APACHE II score, lower Glasgow coma scale (GCS) score, lower
Table 1 Patients with and without delirium

\begin{tabular}{|c|c|c|c|}
\hline Variables & $\begin{array}{l}\text { No delirium } \\
N=887\end{array}$ & $\begin{array}{l}\text { Delirium } \\
N=196\end{array}$ & $p$ \\
\hline Age, years & $69(61-77)$ & $78(71-84)$ & $<0.01$ \\
\hline Female/male & $261 / 626$ & $49 / 147$ & 0.22 \\
\hline APACHE II score & $14(12-17)$ & $17(14-20)$ & $<0.01$ \\
\hline \multicolumn{4}{|l|}{ Reasons for NIV } \\
\hline COPD exacerbation & $477(53.8 \%)$ & $107(54.6 \%)$ & 0.67 \\
\hline Pneumonia & $184(20.7 \%)$ & $48(24.5 \%)$ & \\
\hline ARDS & $63(7.1 \%)$ & $11(5.6 \%)$ & \\
\hline Pulmonary cancer & $49(5.5 \%)$ & $11(5.6 \%)$ & \\
\hline Interstitial lung disease & $22(2.5 \%)$ & $6(3.1 \%)$ & \\
\hline Asthma & $14(1.6 \%)$ & $1(0.5 \%)$ & \\
\hline Heart failure & $10(1.1 \%)$ & $2(1.0 \%)$ & \\
\hline $\mathrm{OHS}$ & $11(1.2 \%)$ & $2(1.0 \%)$ & \\
\hline Pulmonary embolism & $15(1.7 \%)$ & $0(0 \%)$ & \\
\hline Others & $42(4.7 \%)$ & $8(4.1 \%)$ & \\
\hline \multicolumn{4}{|l|}{ Underlying disease } \\
\hline Hypertension & $335(37.8 \%)$ & $86(43.9 \%)$ & 0.12 \\
\hline Diabetes mellitus & $180(20.3 \%)$ & $51(26.0 \%)$ & 0.08 \\
\hline Chronic heart disease & $163(18.4 \%)$ & $47(24.0 \%)$ & 0.09 \\
\hline Chronic liver disease & $36(4.1 \%)$ & $4(2.0 \%)$ & 0.21 \\
\hline Chronic kidney disease & $30(3.4 \%)$ & $11(5.6 \%)$ & 0.15 \\
\hline \multicolumn{4}{|c|}{ Variables collected before NIV } \\
\hline GCS & $15(15-15)$ & $15(14-15)$ & $<0.01$ \\
\hline $\mathrm{HR}$, beats/min & $111 \pm 23$ & $112 \pm 22$ & 0.92 \\
\hline $\mathrm{RR}$, breaths/min & $30(26-35)$ & $30(26-35)$ & 0.75 \\
\hline MAP, $\mathrm{mmHg}$ & $98(87-111)$ & $92(83-106)$ & $<0.01$ \\
\hline $\mathrm{pH}$ & $7.37(7.29-7.45)$ & $7.34(7.26-7.44)$ & $<0.01$ \\
\hline $\mathrm{PaCO}_{2}, \mathrm{mmHg}$ & $57(35-77)$ & $58(37-81)$ & 0.20 \\
\hline $\mathrm{PaO}_{2} / \mathrm{FiO}_{2}, \mathrm{mmHg}$ & $167(127-224)$ & $168(126-243)$ & 0.71 \\
\hline \multicolumn{4}{|l|}{ Outcomes } \\
\hline NIV failure & $186(21.0 \%)$ & $74(37.8 \%)$ & $<0.01$ \\
\hline NIV duration, days & $3.7(1.7-5.9)$ & $6.3(2.7-11.0)$ & $<0.01$ \\
\hline ICU LOS, days & $6.0(3.8-9.2)$ & $9.0(4.8-15.2)$ & $<0.01$ \\
\hline Hospital LOS, days & $11.0(6.8-17.0)$ & $14.5(7.1-21.1)$ & $<0.01$ \\
\hline ICU mortality & $127(14.3 \%)$ & 65 (33.2\%) & $<0.01$ \\
\hline Hospital mortality & $151(17.0 \%)$ & $73(37.2 \%)$ & $<0.01$ \\
\hline
\end{tabular}

NIV noninvasive ventilation, COPD chronic obstructive pulmonary disease, ARDS acute respiratory disease syndrome, $\mathrm{OHS}$ obesity hypoventilation syndrome, GCS Glasgow coma scale, $H R$ heart rate, $R R$ respiratory rate, MAP mean arterial pressure, LOS length of stay

mean arterial pressure, and lower $\mathrm{pH}$. Furthermore, they had higher NIV failure rates, higher ICU mortality, and higher hospital mortality. They also spent longer time on NIV and stayed longer in the ICU and in the hospital (Additional file 3: Fig. S1).

Delirium was independently associated with NIV failure (odds ratio $[\mathrm{OR}]=1.97,95 \%$ confidence interval $[\mathrm{CI}]$ : $1.34-2.88)$, ICU mortality (OR $=2.58,95 \% \mathrm{CI}: 1.74-3.84)$, and hospital mortality $(\mathrm{OR}=2.55,95 \% \mathrm{CI}: 1.74-3.75)$ 
(Table 2). Bootstrap shows that the OR was 1.97 (95\%CI: 1.34-2.97) for NIV failure, 2.58 (1.64-4.08) for ICU mortality, and 2.55 (1.71-3.93) for hospital mortality. These results were also confirmed in patients with and without COPD. Cox regression analyses also showed that delirium was independently associated with NIV failure (Additional files 1, 2: Table S1, S2).

Among the delirious patients, 51 (26\%) developed hyperactive delirium, 123 (63\%) developed hypoactive delirium, and $22(11 \%)$ developed mixed delirium (Table 3). There were no differences in NIV failure, ICU mortality, or hospital mortality among the three groups. Patients with hyperactive delirium had the lowest NIV duration, ICU stay, and hospital stay. However, all three were higher in patients with hypoactive delirium and much higher in those with mixed delirium (Table 3 and Fig. 1).
Age $(\mathrm{OR}=1.06,95 \% \mathrm{CI}: 1.04-1.08)$ and APACHE II score $(\mathrm{OR}=1.07,95 \% \mathrm{CI}$ : $1.03-1.13)$ were risk factors for delirium, and GCS score $(\mathrm{OR}=0.67,95 \% \mathrm{CI}: 0.56-0.79)$ and mean arterial pressure $(\mathrm{OR}=0.99,95 \% \mathrm{CI}: 0.98-1.00)$ were protective factors (Table 4). However, in patients without COPD, only age and GCS were associated with delirium. The distribution of delirium by age and GCS score is presented in Fig. 2. Patients older than 75 years and with a GCS score less than 14 had the highest incidence of delirium. And patients with MAP less than $85 \mathrm{mmHg}$ had higher proportion of delirium than those with MAP more than $85 \mathrm{mmHg}$ (Additional file 4: Fig. S2).

Table 2 Results of multivariate analyses of risk factors for NIV failure, ICU mortality, and hospital mortality

\begin{tabular}{|c|c|c|c|c|c|c|}
\hline \multirow[t]{2}{*}{ Variables } & \multicolumn{2}{|l|}{ Overall cohort } & \multicolumn{2}{|l|}{ COPD cohort } & \multicolumn{2}{|l|}{ Non-COPD cohort } \\
\hline & OR $(95 \% \mathrm{Cl})$ & $p$ & OR $(95 \% \mathrm{Cl})$ & $p$ & OR $(95 \% \mathrm{Cl})$ & $p$ \\
\hline \multicolumn{7}{|c|}{ Risk factors for NIV failure } \\
\hline Delirium & $1.97(1.34-2.88)$ & $<0.01$ & $2.33(1.27-4.30)$ & $<0.01$ & $1.84(1.13-2.99)$ & 0.02 \\
\hline APACHE II score & $1.08(1.03-1.12)$ & $<0.01$ & $1.09(1.02-1.17)$ & 0.02 & $1.10(1.05-1.15)$ & $<0.01$ \\
\hline GCS & $0.78(0.66-0.91)$ & $<0.01$ & - & - & - & - \\
\hline $\mathrm{RR}$, breaths/min & $1.03(1.00-1.05)$ & 0.02 & - & - & - & - \\
\hline $\mathrm{PaCO}_{2}, \mathrm{mmHg}$ & $0.975(0.968-0.982)$ & $<0.01$ & - & - & - & - \\
\hline $\mathrm{PaO}_{2} / \mathrm{FiO}_{2}, \mathrm{mmHg}$ & $0.996(0.993-0.998)$ & $<0.01$ & - & - & 0.995 (0.992-0.998) & $<0.01$ \\
\hline Age, years & - & - & $1.04(1.01-1.08)$ & $<0.01$ & - & - \\
\hline $\mathrm{HR}$, beats/min & - & - & $1.01(1.00-1.03)$ & 0.04 & - & - \\
\hline Diabetes mellitus & - & - & - & - & $0.57(0.36-0.91)$ & 0.02 \\
\hline \multicolumn{7}{|c|}{ Risk factors for ICU mortality } \\
\hline Delirium & $2.58(1.74-3.84)$ & $<0.01$ & $3.64(1.89-7.01)$ & $<0.01$ & $2.16(1.31-3.56)$ & $<0.01$ \\
\hline APACHE II score & $1.06(1.01-1.11)$ & 0.01 & $1.18(1.09-1.28)$ & $<0.01$ & $1.06(1.01-1.11)$ & 0.03 \\
\hline GCS & $0.77(0.65-0.91)$ & $<0.01$ & - & - & - & - \\
\hline RR, breaths/min & $1.04(1.01-1.06)$ & $<0.01$ & - & - & $1.03(1.00-1.06)$ & 0.03 \\
\hline $\mathrm{PaCO}_{2}, \mathrm{mmHg}$ & $0.978(0.970-0.985)$ & $<0.01$ & - & - & - & - \\
\hline $\mathrm{PaO}_{2} / \mathrm{FiO}_{2}$ & $0.998(0.995-1.000)$ & 0.04 & - & - & $0.997(0.994-1.000)$ & 0.04 \\
\hline Age, years & - & - & $1.04(1.01-1.08)$ & 0.03 & - & - \\
\hline \multicolumn{7}{|c|}{ Risk factors for hospital mortality } \\
\hline Delirium & $2.55(1.74-3.75)$ & $<0.01$ & $3.34(1.81-6.18)$ & $<0.01$ & $2.11(1.29-3.45)$ & $<0.01$ \\
\hline APACHE II score & $1.08(1.03-1.13)$ & $<0.01$ & $1.09(1.02-1.17)$ & 0.02 & $1.09(1.04-1.14)$ & $<0.01$ \\
\hline $\mathrm{HR}$, beats/min & $1.01(1.00-1.02)$ & 0.05 & $1.02(1.00-1.03)$ & 0.01 & - & - \\
\hline RR, breaths/min & $1.03(1.00-1.05)$ & 0.03 & - & - & $1.03(1.00-1.06)$ & 0.02 \\
\hline GCS & $0.82(0.67-0.97)$ & 0.02 & - & - & - & - \\
\hline $\mathrm{PaCO}_{2}, \mathrm{mmHg}$ & $0.975(0.968-0.983)$ & $<0.01$ & - & - & - & - \\
\hline $\mathrm{PaO}_{2} / \mathrm{FiO}_{2}$ & - & - & - & - & $0.997(0.995-1.000)$ & 0.05 \\
\hline Age, years & - & - & $1.06(1.03-1.10)$ & $<0.01$ & - & - \\
\hline
\end{tabular}

$\mathrm{NIV}=$ noninvasive ventilation, $\mathrm{OR}=$ odds ratio, $\mathrm{Cl}=$ confidence internal, $\mathrm{GCS}=\mathrm{Glasgow}$ coma scale, $\mathrm{HR}=$ heart rate, $\mathrm{RR}=$ respiratory rate, $\mathrm{COPD}=\mathrm{chronic}$ obstructive pulmonary disease

Delirium, sex, age, underlying disease, APACHE II score, GCS scores, heart rate, respiratory rate, $\mathrm{pH}, \mathrm{PaCO}_{2}$, and $\mathrm{PaO}_{2} / \mathrm{FiO}_{2}$ were entered into multivariate analyses to identify independent risk factors for NIV failure, ICU mortality, and hospital mortality 
Table 3 Results of subgroup analyses in patients with different subtypes of delirium

\begin{tabular}{|c|c|c|c|c|}
\hline Variables & $\begin{array}{l}\text { Hyperactive delirium } \\
\mathrm{N}=51\end{array}$ & $\begin{array}{l}\text { Hypoactive delirium } \\
\mathrm{N}=123\end{array}$ & $\begin{array}{l}\text { Mixed delirium } \\
\mathrm{N}=22\end{array}$ & $P$ \\
\hline NIV failure & $19(37.3 \%)$ & 47 (38.2\%) & $8(36.4 \%)$ & 0.98 \\
\hline ICU mortality & $16(31.4 \%)$ & $41(33.3 \%)$ & $8(36.4 \%)$ & 0.92 \\
\hline Hospital mortality & $17(33.3 \%)$ & 47 (38.2\%) & $9(40.9 \%)$ & 0.78 \\
\hline NIV duration, days & $3.4(1.7-8.8)$ & $6.5(2.9-11.0)$ & $10.1(6.3-20.5)$ & $<0.01$ \\
\hline ICU LOS, days & $6.8(3.3-14.1)$ & $8.9(5.0-15.1)$ & $12.3(8.1-21.2)$ & $<0.01$ \\
\hline Hospital LOS, days & $12.9(5.8-19.0)$ & $15.0(7.9-21.0)$ & $19.5(9.5-31.0)$ & 0.03 \\
\hline Delirium days & $1(1-2)$ & $1(1-3)$ & $7(3-12)$ & $<0.01$ \\
\hline
\end{tabular}

NIV = noninvasive ventilation, $\mathrm{LOS}=$ length of stay
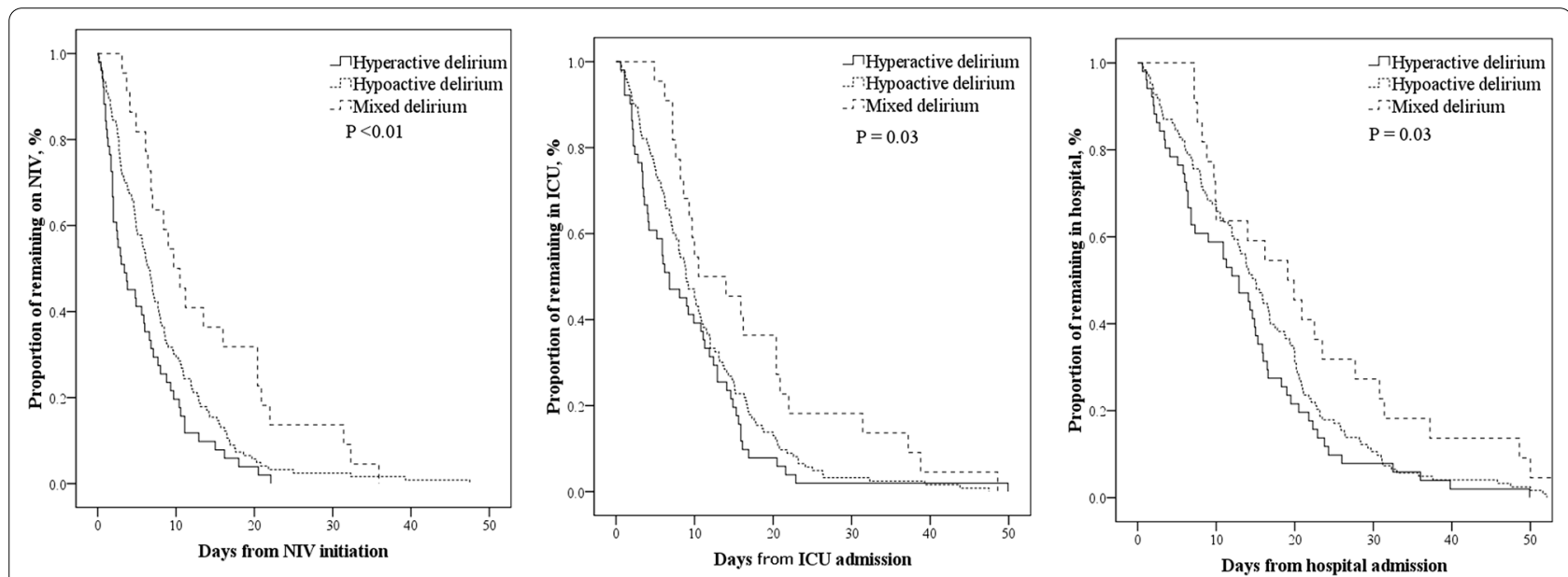

Fig. 1 Resource use among patients with hyperactive, hypoactive, and mixed delirium

Table 4 Results of multivariate analyses of risk factors for delirium

\begin{tabular}{llllll}
\hline Variables & Overall cohort & & COPD cohort & & Non-COPD cohort \\
\hline & OR $(95 \% \mathrm{Cl})$ & $p$ & OR $(95 \% \mathrm{Cl})$ & $p$ & OR $(95 \% \mathrm{Cl})$ \\
Age, years & $1.06(1.04-1.08)$ & $<0.01$ & $1.06(1.03-1.09)$ & $<0.01$ & $1.07(1.05-1.09)$ \\
GCS & $0.67(0.56-0.79)$ & $<0.01$ & $0.74(0.60-0.92)$ & $<0.01$ & $0.48(0.37-0.63)$ \\
APACHE II score & $1.07(1.03-1.13)$ & $<0.01$ & $1.08(1.00-1.15)$ & 0.04 & - \\
MAP & $0.99(0.98-1.00)$ & 0.01 & $0.98(0.97-0.99)$ & $<0.01$ & - \\
\hline
\end{tabular}

$\mathrm{NIV}=$ noninvasive ventilation, $\mathrm{OR}=$ odds ratio, $\mathrm{Cl}=$ confidence internal, $\mathrm{GCS}=\mathrm{Glasgow}$ coma scale, $\mathrm{RR}=$ respiratory rate, $\mathrm{MAP}=$ mean arterial pressure, $\mathrm{COPD}=$ chronic obstructive pulmonary disease

Reasons for NIV, sex, age, underlying disease, APACHE II score, GCS, heart rate, respiratory rate, $\mathrm{pH}, \mathrm{PaCO}_{2}$, and $\mathrm{PaO}_{2} / \mathrm{FiO}_{2}$ were entered into multivariate analyses to identify independent risk factors for delirium

\section{Discussion}

To the best of our knowledge, this is the largest study of the incidence, characteristics, and outcomes of delirium in NIV patients. A systematic review and meta-analysis investigated 239 NIV patients for incidence of delirium [21]. Its data were extracted from three studies published before 2012 [22-24]. One of those only enrolled COPD patients $>65$ years old and did not report how delirium was assessed [22]. In another, delirium was considered if moderate or pronounced encephalopathy was diagnosed [23]. Only one of those three included an assessment of delirium using CAM-ICU (a recommended tool for diagnosis of delirium); however, it only enrolled hypercapnic patients with a median age of 82 years [24]. For these reasons, the results of that systematic review [22] are inaccurate. Another study was published in 2016 [25], which 


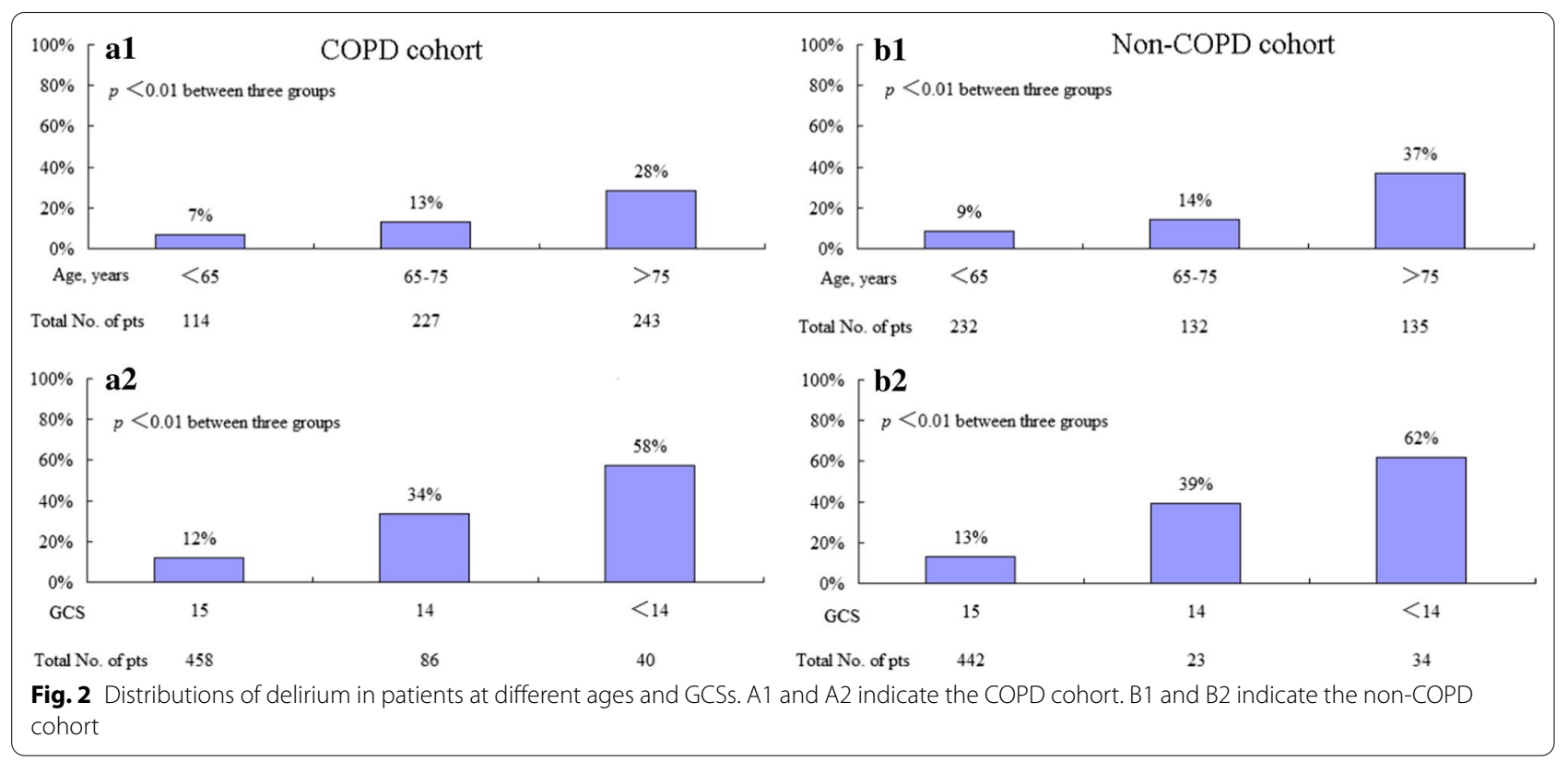

enrolled 153 hypercapnic patients but only reported an association between delirium and 1-year mortality. Due to the small sample size, the relationship among the subtypes of delirium was not reported [21-25]. In our study, we enrolled 1083 NIV patients with different ages and diagnoses. The large sample size enabled us to perform stratification analyses based on diagnosis and age and further explore the relationships among subtypes of delirium.

Hypoactive delirium was the most common subtype reported by Krewulak et al. [2]. In another study, hyperactive delirium was the most common subtype [26]. In our study, hypoactive delirium was the most common subtype, followed by hyperactive delirium and then mixed delirium. In addition, patients with mixed delirium had the largest number of delirium days and the longest ICU stays. To the best of our knowledge, this is the first study to report a distribution among delirium subtypes in NIV patients. Thus, it adds something new to the field that can help clinical practitioners manage NIV patients. For instance, it can reinforce that the incidence of delirium in NIV patients is not low, and delirium is associated with poor outcomes. Awareness of this may lead staff to pay closer more attention to the diagnosis, prevention, and treatment of delirium in NIV patients.

Age, level of consciousness, and disease severity are recognized risk factors for delirium [27-29]. Our study supports this. However, unlike previous studies, we found that mean arterial pressure was a protective factor for delirium. Patients with lower mean arterial pressure were more likely to experience delirium during NIV intervention. Low mean arterial pressure may decrease cerebral blood flow perfusion and lead to neuropsychic symptoms and thus cause delirium. However, this is only a hypothesis, and further exploration is required.

Many studies have found that disease severity, low level of consciousness, increased respiratory rate, and decreased oxygenation are associated with NIV failure [30, 31]. Our study also confirms this. Unlike previous studies, we found that delirium is independently associated with NIV failure both in patients with and without COPD. This provides clinical practitioners with novel insight for understanding NIV failure.

Delirium is highly associated with ICU and hospital mortality $[8,10-12]$. Our study supports this in an NIV population. Because delirium patients often have a poor prognosis, it is necessary to prevent delirium. Pain relief, early mobilization, improvement of sleep quality, and minimal noise are promising methods for reducing delirium in NIV patients [32]. Based on the results found in this study, keeping the MAP more than $85 \mathrm{mmHg}$ is a promising strategy to reduce delirium. Further study is encouraged to confirm this issue.

Our study had several limitations. First, it was a single-center observational study performed in an ICU. Because we only enrolled medical patients, the incidence of delirium may have been skewed. Second, we only enrolled patients in whom NIV was used as a firstline intervention. Those who received NIV followed by high-flow nasal cannula or invasive mechanical ventilation were excluded. This means that our results cannot be extrapolated to the total NIV population. Third, we 
only assessed delirium every morning from NIV initiation to termination. It may be that some patients who were delirious before the start of NIV crossed over in to the non-delirious group if they demonstrated no symptoms of delirium during NIV. Therefore, the incidence of delirium may have been underestimated. Fourth, it was impossible to obtain daily sequential organ failure assessment (SOFA) scores because some of the variables used to calculate SOFA scores were unavailable. Because daily SOFA is superior to APACHE II in terms of disease severity, our use of APACHE II may have reduced the accuracy of our measurements of disease severity.

\section{Conclusion}

The incidence of delirium in the NIV population is high. Hypoactive delirium is the most common subtype in this population. Delirium is associated with increased likelihood of poor outcomes (NIV failure, ICU mortality, and hospital mortality) and use of medical resources (duration of NIV, LOS in the ICU, and LOS in the hospital).

\section{Abbreviations}

NIV: Noninvasive ventilation; ICU: Intensive care unit; COPD: Chronic obstructive pulmonary disease; ARDS: Acute respiratory distress syndrome; $\mathrm{OHS}$ : Obesity hypoventilation syndrome; GCS: Glasgow coma scale; HR: Heart rate; RR: Respiratory rate; MAP: Mean arterial pressure; LOS: Length of stay; OR: Odds ratio; Cl: Confidence interval; CAM-ICU: Confusion Assessment Method for the ICU; RASS: Richmond Agitation-Sedation Scale.

\section{Supplementary Information}

The online version contains supplementary material available at https://doi. org/10.1186/s12890-021-01517-3.

Additional file 1. Supplementary Table 1. Results of Cox regression analyses for NIV failure.

Additional file 2. Supplementary Table 2. Results of Cox regression analyses for NIV failure, ICU mortality, and hospital mortality among patients with pneumonia/ARDS.

Additional file 3. Supplementary Figure 1. Resource use among patients with and without delirium.

Additional file 4. Supplementary Figure 2. Distribution of delirium in patients with MAP more than and less than $85 \mathrm{mmHg}$.

Acknowledgements

We thank all the staffs in ICUs to participate in data collection.

\section{Authors' contributions}

JD conceived the study, joined in study design, study management, data collection, data analyses, and manuscript revision. RZ participated in study design, study management, data collection and drafted the manuscript. LB, $\mathrm{XH}, \mathrm{SH}$ and LZ participated in study design and data collection, and revised the manuscript. All authors read and approved the final version.

\section{Funding}

This study was supported by the National Natural Science Foundation of China (Grant No. 81700015) and by Cultivating Fund of the First Affiliated
Hospital of Chongqing Medical University (Grant No. PYJJ2017-20). The founders had no role in study design, data collection and analysis, decision to publish, or preparation of the manuscript.

\section{Availability of data and materials}

The datasets analyzed during the current study available from the corresponding author on reasonable request.

\section{Declarations}

\section{Consent for publication}

All authors have reviewed and approved the manuscript for publication.

\section{Ethical approval and consent to participate}

The Institutional Review Board of the First Affiliated Hospital of Chongqing Medical University approved the study protocol. Informed consent was waived as the observational nature.

\section{Competing interests}

We declare that we have no competing interests.

Received: 16 August 2020 Accepted: 28 April 2021

Published online: 11 May 2021

\section{References}

1. Barr J, Fraser GL, Puntillo K, et al. Clinical practice guidelines for the management of pain, agitation, and delirium in adult patients in the intensive care unit. Crit Care Med. 2013;41:263-306.

2. Krewulak KD, Stelfox HT, Leigh JP, et al. Incidence and prevalence of delirium subtypes in an adult ICU: a systematic review and meta-analysis. Crit Care Med. 2018;46:2029-35.

3. Hayhurst CJ, Pandharipande PP, Hughes CG. Intensive care unit delirium: a review of diagnosis, prevention, and treatment. Anesthesiology. 2016;125:1229-41.

4. Pandharipande PP, Ely EW, Arora RC, et al. The intensive care delirium research agenda: a multinational, interprofessional perspective. Intensive Care Med. 2017:43:1329-39.

5. Knauert MP, Gilmore EJ, Murphy TE, et al. Association between death and loss of stage N2 sleep features among critically III patients with delirium. J Crit Care. 2018:48:124-9.

6. Devlin JW, Skrobik Y, Gelinas C, et al. Clinical practice guidelines for the prevention and management of pain, agitation/sedation, delirium, immobility, and sleep disruption in adult patients in the ICU. Crit Care Med. 2018:46:e825-73.

7. Grahl JJ, Stollings JL, Rakhit S, et al. Antimicrobial exposure and the risk of delirium in critically ill patients. Crit Care. 2018;22:337.

8. Salluh J, Wang H, Schneider EB, et al. Outcome of delirium in critically ill patients: systematic review and meta-analysis. BMJ 2015; 350:h2538.

9. Ely EW, Inouye SK, Bernard GR, et al. Delirium in mechanically ventilated patients: validity and reliability of the confusion assessment method for the intensive care unit (CAM-ICU). JAMA. 2001;286:2703-10.

10. Kim S, Kim JJ, Oh J, et al. Delirium characteristics and outcomes in medical and surgical Inpatients: a subgroup analysis. J Crit Care. 2018;43:156-62.

11. Shehabi Y, Riker RR, Bokesch PM, et al. Delirium duration and mortality in lightly sedated, mechanically ventilated intensive care patients. Crit Care Med. 2010;38:2311-8.

12. Milbrandt EB, Deppen $\mathrm{S}$, Harrison $\mathrm{PL}$, et al. Costs associated with delirium in mechanically ventilated patients. Crit Care Med. 2004;32:955-62.

13. Su X, Meng ZT, Wu XH, et al. Dexmedetomidine for prevention of delirium in elderly patients after non-cardiac surgery: a randomised, double-blind, placebo-controlled trial. Lancet. 2016;388:1893-902.

14. van den Boogaard M, Slooter AJC, Bruggemann RJM, et al. Effect of haloperidol on survival among critically III adults with a high risk of delirium: the REDUCE Randomized Clinical Trial. JAMA. 2018;319:680-90.

15. Skrobik Y, Duprey MS, Hill NS, et al. Low-dose nocturnal dexmedetomidine prevents ICU delirium. A randomized, placebo-controlled trial. Am J Respir Crit Care Med. 2018;197:1147-56. 
16. Fan L, Zhao Q, Liu Y, et al. Semiquantitative cough strength score and associated outcomes in noninvasive positive pressure ventilation patients with acute exacerbation of chronic obstructive pulmonary disease. Respir Med. 2014;108:1801-7.

17. Duan J, Han X, Bai L, et al. Assessment of heart rate, acidosis, consciousness, oxygenation, and respiratory rate to predict noninvasive ventilation failure in hypoxemic patients. Intensive Care Med. 2017:43:192-9.

18. Duan J, Tang X, Huang S, et al. Protocol-directed versus physiciandirected weaning from noninvasive ventilation: the impact in chronic obstructive pulmonary disease patients. J Trauma Acute Care Surg. 2012;72:1271-5.

19. Sessler CN, Gosnell MS, Grap MJ, et al. The Richmond Agitation-Sedation Scale: validity and reliability in adult intensive care unit patients. Am J Respir Crit Care Med. 2002;166:1338-44.

20. Robinson TN, Raeburn CD, Tran ZV, et al. Motor subtypes of postoperative delirium in older adults. Arch Surg. 2011;146:295-300.

21. Charlesworth M, Elliott MW, Holmes JD. Noninvasive positive pressure ventilation for acute respiratory failure in delirious patients: understudied, underreported, or underappreciated? A systematic review and metaanalysis. Lung. 2012:190:597-603.

22. Rozzini R, Sabatini T, Trabucchi M. Non-invasive ventilation for respiratory failure in elderly patients. Age Ageing. 2006;35:546-7.

23. Carlucci A, Richard JC, Wysocki M, et al. Noninvasive versus conventional mechanical ventilation. An epidemiologic survey. Am J Respir Crit Care Med. 2001;163:874-80.

24. Roche Campo F, Drouot $X$, Thille AW, et al. Poor sleep quality is associated with late noninvasive ventilation failure in patients with acute hypercapnic respiratory failure. Crit Care Med. 2010;38:477-85.
25. Chan KY, Cheng LS, Mak IW, et al. Delirium is a strong predictor of mortality in patients receiving non-invasive positive pressure ventilation. Lung. 2017:195:115-25.

26. Gual N, Inzitari M, Carrizo G, et al. Delirium subtypes and associated characteristics in older patients with exacerbation of chronic conditions. Am J Geriatr Psychiatry. 2018;26:1204-12.

27. Janssen TL, Hosseinzoi E, Vos DI, et al. The importance of increased awareness for delirium in elderly patients with rib fractures after blunt chest wall trauma: a retrospective cohort study on risk factors and outcomes. BMC Emerg Med. 2019;19:34.

28. Gual N, Morandi A, Perez LM, et al. Risk factors and outcomes of delirium in older patients admitted to postacute care with and without dementia. Dement Geriatr Cogn Disord. 2018;45:121-9.

29. Chaiwat O, Chanidnuan M, Pancharoen W, et al. Postoperative delirium in critically ill surgical patients: incidence, risk factors, and predictive scores. BMC Anesthesiol. 2019;19:39.

30. Ozyilmaz E, Ugurlu AO, Nava S. Timing of noninvasive ventilation failure: causes, risk factors, and potential remedies. BMC Pulm Med. 2014:14:19.

31. Nava S, Hill N. Non-invasive ventilation in acute respiratory failure. Lancet. 2009;374:250-9.

32. Tang B, Wang XT, Chen WJ, et al. Experts consensus on the management of delirium in critically ill patients. Zhonghua Nei Ke Za Zhi. 2019;58:108-18.

\section{Publisher's Note}

Springer Nature remains neutral with regard to jurisdictional claims in published maps and institutional affiliations.
Ready to submit your research? Choose BMC and benefit from:

- fast, convenient online submission

- thorough peer review by experienced researchers in your field

- rapid publication on acceptance

- support for research data, including large and complex data types

- gold Open Access which fosters wider collaboration and increased citations

- maximum visibility for your research: over $100 \mathrm{M}$ website views per year

At BMC, research is always in progress.

Learn more biomedcentral.com/submissions 